\title{
On Rotated Time-Frequency Kernels
}

\author{
Martin J. Bastiaans, Senior Member, IEEE, Tatiana Alieva, and LJubiša Stanković, Senior Member, IEEE
}

\begin{abstract}
The principal axes of the time-frequency representation of a signal are defined as those mutually orthogonal directions in the time-frequency plane for which the width of the signal's fractional power spectrum is minimum or maximum. The time-frequency kernels used in the Cohen class of time-frequency representations are then rotated in the time-frequency plane, in order to align the kernels' preferred axes to the signal's principal axes. It is shown that the resulting time-frequency representations show a better reduction of cross-terms without too severely degrading the autoterms than the corresponding original time-frequency representations.
\end{abstract}

Index Terms-Ambiguity function, fractional Fourier transform, time-frequency signal analysis, Wigner distribution.

\section{INTRODUCTION}

$\mathbf{T}$ O REPRESENT a signal in a time-frequency plane, many time-frequency representations are used nowadays, each with its own advantages and disadvantages. The well-known Wigner distribution (WD) of a multicomponent signal, for instance, shows highly concentrated autoterms, but suffers from cross-terms, which may even hide some of the autoterms. Many other distributions have been proposed to optimally represent the signal in a time-frequency plane, with significantly reduced cross-terms but without a too severe degrading of the autoterms. Such distributions may result from a weighting of the WD by an appropriate time-frequency kernel, leading to Cohen's class of time-frequency distributions.

In this letter, we use kernels from the Cohen class, but we rotate these kernels in the time-frequency plane through an angle that is determined by the characteristics of the signal. In particular, we consider the signal's fractional power spectrum-the squared modulus of the fractional Fourier transform - and find its principal axes, determined by those two orthogonal directions for which the width of the fractional power spectrum is minimum or maximum, respectively. We then show that if a kernel's preferred axes are aligned to these two directions, the resulting time-frequency distribution shows a much better performance with respect to the autoterms concentration and the cross-terms reduction than the distribution that would have re-

Manuscript received February 8, 2002; revised July 24, 2002. This work was supported by the Spanish Ministry of Education, Culture and Sports under Contract SB 2000-0166. The work of LJ. Stankovic was supported by the Volkswagen Stiftung, Germany. The associate editor coordinating the review of this manuscript and approving it for publication was Prof. Dimitris A. Pados.

M. J. Bastiaans is with the Technische Universiteit Eindhoven, Faculteit Elektrotechniek, 5600 MB Eindhoven, The Netherlands (e-mail: m.j.bastiaans@ieee.org).

T. Alieva is with the Universidad Complutense de Madrid, Facultad de Ciencias Físicas, 28040 Madrid, Spain (e-mail: talieva@ fis.ucm.es).

LJ. Stanković is with the University of Montenegro, Elektrotehnicki Fakultet, 81000 Podgorica, Montenegro, Yugoslavia (e-mail: 1.stankovic@ieee.org).

Digital Object Identifier 10.1109/LSP.2002.805118 sulted if the original nonaligned kernel would have been used. The more dominantly the time-frequency content of the signal is located along one principal axis, the better the improvement of the resulting time-frequency representation will be.

The proposed procedure is demonstrated on a multicomponent signal, using some of the well-known Cohen-class kernels.

\section{Wigner Distribution AND THE COHEN ClASS}

Let us start with the WD $W_{x}(t, f)$ of a time signal $x(t)$, defined as

$$
W_{x}(t, f)=\int_{-\infty}^{\infty} x(t+\tau / 2) x^{*}(t-\tau / 2) \exp (-j 2 \pi f \tau) d \tau .
$$

It is well known that the WD of a multicomponent signal suffers from cross-terms. Therefore, a weighted version of the WD is often used as a time-frequency representation of the signal, where the weighting kernel $\Phi(t, f)$ is chosen such that the crossterms are reduced without degrading the autoterms too severely. This leads to the Cohen class of time-frequency representations [1]

$$
C_{x}(t, f)=\int_{-\infty}^{\infty} \int_{-\infty}^{\infty} \Phi\left(t_{o}, f_{o}\right) W_{x}\left(t-t_{o}, f-f_{o}\right) d t_{o} d f_{o} .
$$

Many kernels have been proposed during the past decades [2].

In designing a kernel $\Phi(t, f)$, it is advantageous to go from the Wigner domain to the ambiguity domain by means of a Fourier/inverse Fourier transformation (FIFT). In that case, the FIFT $\bar{C}_{x}(\tau, \nu)$ of the time-frequency representation $C_{x}(t, f)$

$$
\bar{C}_{x}(\tau, \nu)=\int_{-\infty}^{\infty} \int_{-\infty}^{\infty} C_{x}(t, f) \exp [-j 2 \pi(\nu t-f \tau)] d t d f
$$

is related to the FIFT $\bar{\Phi}(\tau, \nu)$ of the kernel $\Phi(t, f)$ and the ambiguity function (AF) $A_{x}(\tau, \nu)$

$$
A_{x}(\tau, \nu)=\int_{-\infty}^{\infty} x(t+\tau / 2) x^{*}(t-\tau / 2) \exp (-j 2 \pi \nu t) d t
$$

by a simple product relation

$$
\bar{C}_{x}(\tau, \nu)=\bar{\Phi}(\tau, \nu) A_{x}(\tau, \nu) .
$$

We remark that the AF is the FIFT of the WD

$$
A_{x}(\tau, \nu)=\int_{-\infty}^{\infty} \int_{-\infty}^{\infty} W_{x}(t, f) \exp [-j 2 \pi(\nu t-f \tau)] d t d f
$$

and that the moments of the WD are related to the derivatives of the $\mathrm{AF}$ as

$$
\begin{aligned}
\int_{-\infty}^{\infty} \int_{-\infty}^{\infty} W_{x}(t, f) t^{n} f^{m} d t d f & =\left.\frac{(-1)^{n}}{(j 2 \pi)^{m+n}} \frac{\partial^{m+n} A_{x}(\tau, \nu)}{\partial \tau^{m} \partial \nu^{n}}\right|_{\tau=\nu=0}
\end{aligned}
$$


As examples of time-frequency kernels for some well-known time-frequency distributions, we mention [2]
- Wigner

- Born-Jordan

- Zhao-Atlas-Marks (cone)

- Butterworth

- Choi-Williams (exponential)

- generalized exponential

$$
\left|X_{\alpha}(t)\right|^{2}=\int_{-\infty}^{\infty} A_{X_{\alpha}}(0, \nu) \exp (j 2 \pi \nu t) d \nu
$$

1 $\frac{\sin (\pi \tau \nu)}{\pi \tau \nu}$ $g(\tau)|\tau| \frac{\sin (\pi \tau \nu)}{\pi \tau \nu}$ $1 /\left[1+\left(\frac{\tau}{\tau_{o}}\right)^{2 M}\left(\frac{\nu}{\nu_{o}}\right)^{2 N}\right]$ $\exp \left[-\frac{(2 \pi \tau \nu)^{2}}{\sigma}\right]$ $\exp \left[-\left(\frac{\tau}{\tau_{o}}\right)^{2 M}\left(\frac{\nu}{\nu_{o}}\right)^{2 N}\right]$ where the Choi-Williams kernel is a special case of the generalized exponential kernel with $M=N=1$ and $\sigma=\left(2 \pi \tau_{o} \nu_{o}\right)^{2}$.

In many cases, the kernel $\Phi(t, f)$ [and $\bar{\Phi}(\tau, \nu)$ ] shows a preferred behavior in the time and/or the frequency direction. The degree of cross-term reduction (and degrading of the autoterms) then depends on the way in which the WD is oriented in the time-frequency plane. If the orientation is along the time and/or the frequency direction, the kernel may act as expected; in the case of a different orientation, the effect of the kernel is not optimal [3]. We suggest, therefore, to rotate the kernel in such a way that its preferred axes coincide with the principal axes of the WD (and the AF). Note that, although the rotated distributions may not satisfy the common marginal properties, they satisfy generalized ones [4].

\section{FRACTIONAL Fourier TRANSFORM}

To find the principal axes of the WD (and the AF), we introduce the fractional Fourier transform (FT) $X_{\alpha}(u)$ of the signal $x(t)$, defined by [5]

$$
X_{\alpha}(u)=\int_{-\infty}^{\infty} K_{\alpha}(t, u) x(t) d t
$$

where the kernel $K_{\alpha}(t, u)$ is given by

$$
K_{\alpha}(t, u)=\frac{\exp \left(j \frac{1}{2} \alpha\right)}{\sqrt{j \sin \alpha}} \exp \left(j \pi \frac{\left(t^{2}+u^{2}\right) \cos \alpha-2 u t}{\sin \alpha}\right) .
$$

Note in particular that $X_{0}(u)=x(u)$ and $X_{\pi}(u)=x(-u)$, and $X_{\pi / 2}(u)$ corresponds to a normal FT.

It is well known (e.g., see [5], [6], or [7]) that the fractional FT corresponds to a rotation of the WD in the $(t, f)$ plane, as well as to a rotation of the $\mathrm{AF}$ in the $(\tau, \nu)$ plane

$$
\begin{aligned}
& W_{X_{\alpha}}(t, f)=W_{x}(t \cos \alpha-f \sin \alpha, t \sin \alpha+f \cos \alpha) \\
& A_{X_{\alpha}}(\tau, \nu)=A_{x}(\tau \cos \alpha-\nu \sin \alpha, \tau \sin \alpha+\nu \cos \alpha) .
\end{aligned}
$$

If we introduce the fractional power spectra $\left|X_{\alpha}(t)\right|^{2}$ as the squared moduli of the corresponding fractional FTs, we have the following relations between these fractional power spectra on the one hand and the WD and the AF on the other:

$$
\left|X_{\alpha}(t)\right|^{2}=\int_{-\infty}^{\infty} W_{X_{\alpha}}(t, f) d f
$$

\section{Moments OF THE Fractional POWER SPeCtra}

We now introduce the moments of the fractional power spectra [8]. For the zero-order moment $E=\int_{-\infty}^{\infty}\left|X_{\alpha}(t)\right|^{2} d t$ we have $E=\int_{-\infty}^{\infty} \int_{-\infty}^{\infty} W_{X_{\alpha}}(t, f) d t d f=A_{X_{\alpha}}(0,0)$ (see (12) and (13), and also (7) with $m=n=0$ ). Note that the zero-order moment represents the signal's energy and that-in accordance with Parseval's theorem for a unitary transformation-it does not depend on $\alpha$.

For the (normalized) first-order moments $m_{\alpha}$, with $m_{\alpha} E=$ $\int_{-\infty}^{\infty}\left|X_{\alpha}(t)\right|^{2} t d t$, we may proceed along the same lines, now chosing $m=0$ and $n=1$ in (7). Note that the first-order moments are related to the centers of gravity of the fractional power spectra and that they satisfy the relationship $m_{\alpha}=m_{0} \cos \alpha+$ $m_{\pi / 2} \sin \alpha$.

For the (normalized) second-order moments $w_{\alpha}$, with $w_{\alpha} E=\int_{-\infty}^{\infty}\left|X_{\alpha}(t)\right|^{2} t^{2} d t$, we have

$$
\begin{aligned}
w_{\alpha} E & =\int_{-\infty}^{\infty} \int_{-\infty}^{\infty} W_{X_{\alpha}}(t, f) t^{2} d t d f \\
& =\left.\frac{1}{(2 \pi j)^{2}} \frac{\partial^{2} A_{X_{\alpha}}(0, \nu)}{\partial \nu^{2}}\right|_{\nu=0}
\end{aligned}
$$

(see (7) with $m=0$ and $n=2$ ). We also introduce the mixed second-order moments of the WD and the mixed second-order derivative of the $\mathrm{AF}$, while defining the (normalized) mixed second-order moments $\mu_{\alpha}$ (see (7) with $m=n=1$ )

$$
\begin{aligned}
\mu_{\alpha} E & =\frac{1}{4 \pi j} \int_{-\infty}^{\infty}\left[\frac{\partial X_{\alpha}(t)}{\partial t} X_{\alpha}^{*}(t)-X_{\alpha}(t) \frac{\partial X_{\alpha}^{*}(t)}{\partial t}\right] t d t \\
& =\int_{-\infty}^{\infty} \int_{-\infty}^{\infty} W_{X_{\alpha}}(t, f) t f d t d f \\
& =\left.\frac{-1}{(2 \pi j)^{2}} \frac{\partial^{2} A_{X_{\alpha}}(\tau, \nu)}{\partial \tau \partial \nu}\right|_{\tau=0, \nu=0} .
\end{aligned}
$$
$\mu_{\alpha}$, we could as well consider the central second-order moments $w_{\alpha}-m_{\alpha}^{2}$ and $\mu_{\alpha}-m_{\alpha} m_{\alpha+\pi / 2}$, which lead to similar expressions as the ones that we find for the normal moments. Note that the central second-order moments are related to the effective widths of the fractional power spectra.

The second-order moments satisfy the relationships

$$
\begin{aligned}
& w_{\alpha}=w_{0} \cos ^{2} \alpha+w_{\pi / 2} \sin ^{2} \alpha+\mu_{0} \sin 2 \alpha \\
& \mu_{\alpha}=-(1 / 2)\left(w_{0}-w_{\pi / 2}\right) \sin 2 \alpha+\mu_{0} \cos 2 \alpha .
\end{aligned}
$$

In general, all second-order moments $w_{\alpha}$ and $\mu_{\alpha}$ can be obtained from any three second-order moments $w_{\alpha}$ taken for three different angles $\alpha$ from the region $[0, \pi)$; we have, for instance, $\mu_{0}=w_{\pi / 4}-(1 / 2)\left(w_{0}+w_{\pi / 2}\right)$. This implies that the corresponding three fractional power spectra define all second-order moments.

From (16), we conclude that the sum of the moments $w_{\alpha}+$ $w_{\alpha+\pi / 2}$ does not depend on $\alpha$

$$
w_{\alpha}+w_{\alpha+\pi / 2}=w_{0}+w_{\pi / 2}
$$

We remark that instead of the second-order moments $w_{\alpha}$ and 


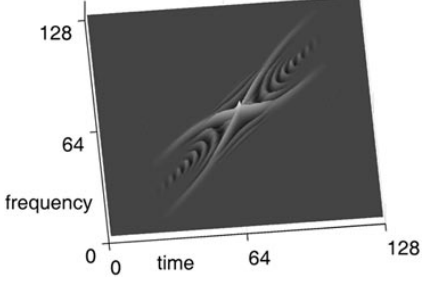

Fig. 1. Wigner distribution of the signal $x(t)$.

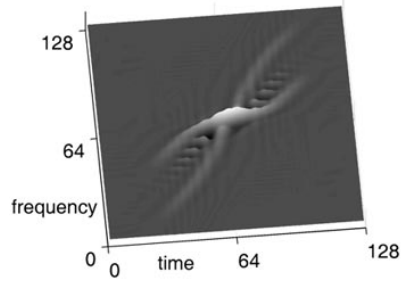

(a)

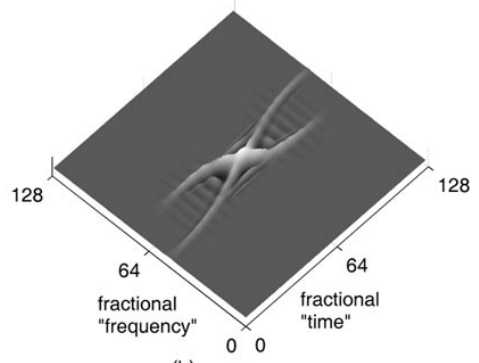

(b)
Fig. 2. Butterworth distribution of the signal $x(t)$ (a) without alignment and (b) with alignment of the kernel.

and from (17) we conclude that

$$
\mu_{\alpha}+\mu_{\alpha+\pi / 2}=0
$$

From (16), it is easy to see that the first derivative of $w_{\alpha}$ with respect to the angle $\alpha$ equals zero for $\alpha=\alpha_{e}$, where $\alpha_{e}$ satisfies the relationship

$$
\tan 2 \alpha_{e}=\frac{2 \mu_{0}}{w_{0}-w_{\pi / 2}}=\frac{2 w_{\pi / 4}-\left(w_{0}+w_{\pi / 2}\right)}{w_{0}-w_{\pi / 2}} .
$$

From the invariance relationship (18), we conclude that $\alpha_{e}$ determines the domain with the smallest $w_{\alpha}$, while $\alpha_{e} \pm \pi / 2$ corresponds to the domain with the largest $w_{\alpha}$ or vice versa. Moreover, from (17), (19), and (20) we also conclude that $\mu_{\alpha_{e}}=$ $\mu_{\alpha_{e} \pm \pi / 2}=0$.

We now identify the direction $\alpha_{e}$ with one of the principal axes, and the direction $\alpha_{e} \pm \pi / 2$ with the other one. If $\Phi(t, f)$ denotes the time-frequency kernel that we use, the rotated version reads $\Phi(t \cos \alpha+f \sin \alpha,-t \sin \alpha+f \cos \alpha)$, and the general Cohen-class time-frequency representation takes the form

$$
\begin{aligned}
& C_{x}(t, f)=\int_{-\infty}^{\infty} \int_{-\infty}^{\infty} W_{x}\left(t-t_{o}, f-f_{o}\right) \\
& \quad \cdot \Phi\left(t_{o} \cos \alpha+f_{o} \sin \alpha,-t_{o} \sin \alpha+f_{o} \cos \alpha\right) d t_{o} d f_{o} .
\end{aligned}
$$

Note that we can also write

$C_{X_{\alpha}}(t, f)=\int_{-\infty}^{\infty} \int_{-\infty}^{\infty} W_{X_{\alpha}}\left(t-t_{o}, f-f_{o}\right) \Phi\left(t_{o}, f_{o}\right) d t_{o} d f_{o}$

\section{EXAMPLES}

As an example, consider the signal

$$
\begin{aligned}
x(t)= & \exp \left[-(3 t)^{8}\right]\left\{\exp \left[j\left(192 \pi t^{2}-\frac{8 \cos (4 \pi t)}{\pi}\right)\right]\right. \\
& \left.+\exp \left[j\left(64 \pi t^{2}+\frac{8 \cos (4 \pi t)}{\pi}\right)\right]\right\} .
\end{aligned}
$$

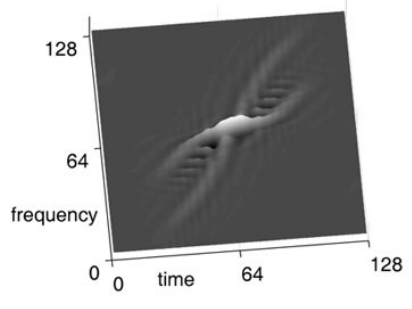

(a)

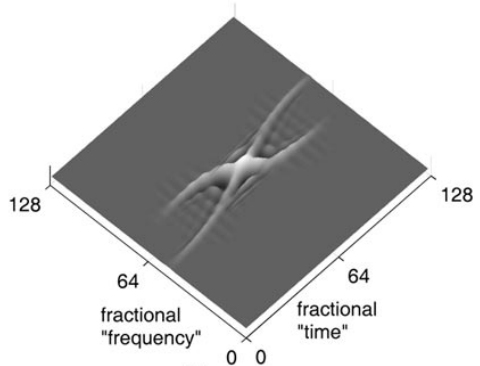

(b)
Fig. 3. Generalized exponential distribution of the signal $x(t)$ (a) without alignment and (b) with alignment of the kernel.

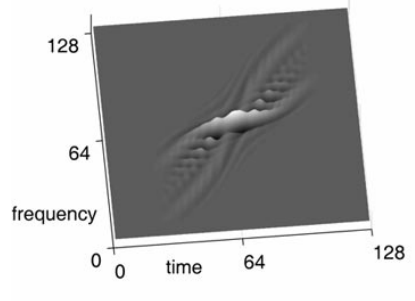

(a)

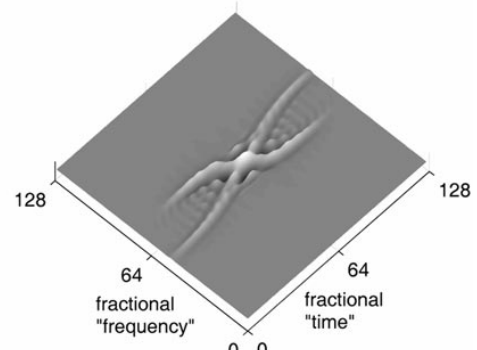

(b)
Fig. 4. Zhao-Atlas-Marks (cone kernel) distribution of the signal $x(t)$ (a) without alignment and (b) with alignment of the kernel.

The values of the normalized central moments are $w_{0}=1$, $w_{\pi / 2}=1.38$, and $w_{\pi / 4}=0.07$. From (20), we get $\alpha_{e}=41^{\circ}$ or $\alpha_{e}=-49^{\circ}$. The second-order moment in the direction $41^{\circ}$ is smaller than in any other direction: $w_{41^{\circ}}=0.057$, while the second-order moment in the orthogonal direction is the largest: $w_{-49^{\circ}}=2.01$.

The WD of this signal has been depicted in Fig. 1. In Figs. 2-4, we have depicted the time-frequency representations that result from weighting this WD with several kernels, without and with alignment of the kernels to the principal axes $\left(41^{\circ}\right.$ and $-49^{\circ}$, respectively) of the WD:

- Fig. 2: Butterworth, with $M=1$ and $N=3$;

- Fig. 3: generalized exponential, with $M=1$ and $N=3$;

- Fig. 4: Zhao-Atlas-Marks (cone), with $g(\tau)=\cos ^{4}(\pi \tau)$. The kernel parameters have been chosen such that for the signal under consideration the corresponding distributions show optimal results.

According to (21) and (22), the three time-frequency distributions from the Cohen class are realized as a weighted WD, where the weighting kernel $\Phi(t, f)$ is the FIFT of $\bar{\Phi}(\tau, \nu)$. A region $-1 / 2 \leq \tau<1 / 2,-128 \pi \leq 2 \pi \nu<128 \pi$ in the ambiguity domain is considered. This corresponds to the time interval $T=1$ and the sampling interval $\Delta t=1 / 128$. In discretization, 128 samples are taken along each ambiguity axis, and the two-dimensional inverse FIFT of the $\bar{\Phi}(\tau, \nu)$-samples is calculated. The matrix $\Phi(n, k)$ obtained in this way is convolved with the WD, according to (21) and (22) (original and rotated, respectively). The size of the obtained Cohen-class distribution is kept the same as the size of the WD, using the command conv2(WD,Kernel,'same') in Matlab. The parameters $\tau_{o}, \nu_{o}$, $M$, and $N$ for the Butterworth and the generalized exponential distribution are $\tau_{o}=0.05,2 \pi \nu_{o}=25.6 \pi, M=1$, and $N=3$, 
while the window $g(\tau)$ in the Zhao-Atlas-Marks distribution is a Hanning window: $g(\tau)=\cos ^{4}(\pi \tau)$.

We conclude that the performance with respect to the autoterms concentration and the cross-terms reduction is better for the aligned kernels than for the nonaligned ones.

Note that we have chosen kernels whose time-behavior is significantly different from the frequency behavior; kernels that depend on the product $\tau \nu$ only-such as the ones that lead to the Born-Jordan, the Choi-Williams (exponential), or the reduced interference distribution, for instance-do not show a clear improvement in cross-term reduction when they are rotated. Such kernels could be used, of course, after having been modified by an additional narrowing window in the $\tau$ direction; cf. the Zhao-Atlas-Marks (cone) kernel, which differs from the Born-Jordan kernel by the additional window $g(\tau)|\tau|$.

We finally remark that we have considered the rotation of standard kernels. The same effect can be achieved by using standard kernels with an additional ambiguity domain axis corresponding to the determined principal axis, and generalizedmarginal distributions [4]. Since, in this way, we introduce an additional rotated axis, the obtained results are very similar with the ones presented in this letter. These results will be presented in one of our next papers.

\section{CONCLUSION}

The fractional power spectrum-i.e., the squared modulus of the fractional FT of a signal - is considered, and the signal's principal axes are defined as the two orthogonal directions for which the fractional power spectrum has a minimum or maximum width. A Cohen-class kernel that is used to smooth the WD is then rotated in the time-frequency plane and oriented such that its preferred axes are aligned to these two directions. It is shown that the resulting time-frequency distribution yields a better cross-term reduction (without a too severe degrading of the autoterms) than the corresponding original distribution.

The better the signal is located along one principal axis, the better the improvement of the resulting time-frequency distribution will be. In the case that there is no clear principal direction, or there is more than one such direction, the procedure described might not yield a clear improvement. The case of multiple principal directions will be a subject for future work.
The method of aligning kernels to the signal's principal axes can be applied to rather arbitrary time-frequency representations. In a forthcoming paper, we will demonstrate its effect on the weighting of the WD using the generalized [9] S-method [10], in which case the kernels in the ambiguity domain and the Wigner domain are given by

$$
\begin{aligned}
& \bar{\Phi}(\tau, \nu)=\int_{-\infty}^{\infty} A_{\gamma}(-\tau+\theta \sin \alpha,-\nu+\theta \cos \alpha) z(\theta) d \theta \\
& \Phi(t, f)=W_{\gamma}(-t,-f) Z(-[t \cos \alpha-f \sin \alpha])
\end{aligned}
$$

respectively where $Z(f)$ is the FT of $z(t)$.

\section{ACKNOWLEDGMENT}

T. Alieva would like to thank the Spanish Ministry of Education, Culture and Sports for financial help.

\section{REFERENCES}

[1] L. Cohen, Time-Frequency Signal Analysis. Englewood Cliffs, NJ: Prentice-Hall, 1995.

[2] F. Hlawatsch and G. F. Boudreaux-Bartels, "Linear and quadratic timefrequency signal representations," IEEE Signal Processing Mag., vol. 9, no. 2, pp. 21-67, Apr. 1992.

[3] A. K. Özdemir and O. Arıkan, "A high resolution time frequency representation with significantly reduced cross-terms," Proc. ICASSP, vol. 2, pp. II693-II696, 2000.

[4] X.-G. Xia, Y. Owechko, B. H. Soffer, and R. M. Matic, "On generalizedmarginal time-frequency distributions," IEEE Trans. Signal Processing, vol. 44, pp. 2882-2886, Nov. 1996.

[5] L. B. Almeida, "The fractional Fourier transform and time-frequency representations," IEEE Trans. Signal Processing, vol. 42, pp. 3084-3091, Nov. 1994.

[6] A. W. Lohmann and B. H. Soffer, "Relationships between the RadonWigner and fractional Fourier transforms," J. Opt. Soc. Amer. A, vol. 11, pp. 1798-1801, 1994.

[7] LJ. Stanković and I. Djurović, "Relationship between the ambiguity function coordinate transformations and the fractional Fourier transform,” Ann. Télécommun., vol. 53, pp. 316-319, 1998.

[8] T. Alieva and M. J. Bastiaans, "On fractional Fourier transform moments," IEEE Signal Process. Lett., vol. 7, pp. 320-323, Nov. 2000.

[9] LJ. Stanković, T. Alieva, and M. J. Bastiaans, "Fractional-Fourier-domain weighted Wigner distribution," Proc. 11th IEEE Signal Processing Workshop on Statistical Signal Processing, pp. 321-324, 2001.

[10] LJ. Stanković, "A method for time-frequency signal analysis," IEEE Trans. Signal Processing, vol. 49, pp. 225-229, Jan. 1994. 